\title{
KARYOTYPE ANALYSIS IN HAPLOID SUGARBEET ${ }^{1}$
}

\author{
L. CISTUE, $*$ I. ROMAGOSA,* T. TSUCHIYA, $\dagger$ AND J. M. LASA* \\ *Estacion Experimental de Aula Dei, Zaragoza, Spain; and †Department of Agronomy, \\ Colorado State University, Fort Collins, Colorado 80523
}

\begin{abstract}
The mitotic chromosome morphology of the sugarbeet was studied in three haploid plants of independent origin. The average length of the nine metaphase chromosomes was $2.39 \mu \mathrm{m}$, varying from 1.97 to 2.78 $\mu \mathrm{m}$. Three chromosomes had median centromeres, two had submedian centromeres, and four had mediansubmedian centromeres. All nine chromosomes may be identified on the basis of their total length and arm ratio when arranged in a specific manner, although there is a considerable risk of reversal-of-order error.
\end{abstract}

\section{Introduction}

The difficulty in identifying the nine individual sugarbeet chromosomes has been a major problem for cytological studies because of their small and similar sizes. In a detailed study of the chromosome morphology in a homozygous diploid sugarbeet, BOSEMARK and BORMOTOV (1971) reported that seven of the nine chromosomes, based on nomenclature suggested by LEVAN et al. (1964), had more or less median centromeres, and the other two had submedian centromeres. They concluded that, except for the satellited chromosomes, the technique did not show any differences useful in identifying the chromosomes. DE JONG and DE Bock (1978) used haploid sugarbeet plants to study orcein- and Giemsa-stained chromosomes. However, because of variation among haploid plants and the small differences measured along the nine chromosomes, they could not construct an idiogram of the haploid complement. These reports indicated the high probability of reversal-of-order errors (MATERN and SIMAK 1968) in the identification of the nine sugarbeet chromosomes.

TSUCHIYA and NAKAMURA (1979) used ice water in the pretreatment of mitotic sugarbeet chromosomes but did not analyze the individual chromosomes. Cistue (1983) also identified the nine individual chromosomes at the mitotic prophase stage, on the basis of the chromomeric pattern of each pair of homologues. Sugarbeet pachytene chromosomes were morphologically identified, and idiograms of all nine chromosomes were developed by Nakamura and TsuchiYa (1982). How-

\footnotetext{
${ }^{1}$ Supported in part by the United States-Spain Program of Cooperation in Science and Technology, under cooperative research project III P 3040. Contribution from the Aula dei Experiment Station, Zaragoza, Spain, and the Department of Agronomy, Colorado State University. Published with the approval of the Director of Colorado State Agricultural Experiment Station as Scientific Series Paper no. 2910.
}

Manuscript received May 1984; revised manuscript received January 1985.

Address for correspondence and reprints: T. TsuchiYA, Department of Agronomy, Colorado State University, Fort Collins, Colorado 80523. ever, these techniques are not easily utilized and, therefore, are of limited use to anyone but a highly experienced cytologist.

The objective of this study was to develop a standard metaphase karyotype for somatic cells using haploid plants. This karyotype would identify the individual sugarbeet chromosomes, more on the basis of the combination of the relative size and centromeric position than on statistical techniques. A positive identification of individual chromosomes would have considerable value for future cytological and cytogenetic studies in sugarbeet.

\section{Material and methods}

Three haploid plants of independent origin were used: One occurred spontaneously in the progeny of a colchicine-treated plant; the other two were produced by crossing diploid male-sterile plants with green hypocotyls and tetraploid fodder beets homozygous for red hypocotyls (BOSEMARK 1971).

Growing root tips were collected in the early morning and pretreated in ice water for $2-4 \mathrm{~h}$. Longer pretreatment made the chromosomes shorter and inadequate for karyotype analysis. Root tips were fixed in freshly prepared ethanol-glacial acetic acid (3:1) solution and were kept in the fixative for ca. $24 \mathrm{~h}$. Longer fixation often resulted in preparations of poor quality.

Two staining and mounting techniques were used alternately with the same results. The first followed closely that of TSUCHIYA and NAKAMURA (1979), but fixed roots were stained in $2 \%$ orcein in $45 \%$ acetic acid for $3-4 \mathrm{~h}$ instead of $0.8 \%$ acetocarmine for 3-4 days. No hydrolysis was needed. The root tip was mounted in a drop of $45 \%$ acetic acid, and the final squash was made after the slide was heated over an alcohol lamp. The second technique was that of BOSEMARK and BORMOTOV (1971). After hydrolysis for $5 \mathrm{~min}$ in $1 \mathrm{~N}$ $\mathrm{HCl}$, root tips were stained in $2 \%$ lactopropionic orcein for at least $30 \mathrm{~min}$. The final squash was made in the same stain.

Selected cells were photographed on $9 \times 12-\mathrm{cm}$ plates $(\times 5,000)$ or on standard $35-\mathrm{mm}$ film $(\times 1,250)$. Measurements were made on prints 
$(\times 20,000)$. To compensate for the variation in chromosome contraction among different cells, relative values were also calculated.

\section{Results}

Four cells from three haploid plants were selected from a set of metaphase plates (figs. 1, 2), and their chromosomes were measured. The length of the satellite on the short arm of chromosome 1 was not included in the measurements since its length varied greatly from cell to cell. Two parameters were estimated: (1) the total length in $\mu \mathrm{m}$, and (2) the arm ratio, calculated as the ratio of the length of the long to the short arm of each chromosome.

The average length of all chromosomes was 2.39 $\mu \mathrm{m}$, ranging from the shortest, $1.97 \mu \mathrm{m}$, to the longest, the nucleolar chromosome, $2.78 \mu \mathrm{m}$. The average length of the satellite was $0.23 \mu \mathrm{m}$. The arm ratio among chromosomes varied from 1.04 to 2.08 (table 1). An idiogram of the sugarbeet haploid complement (fig. 3) shows the smallest and similar sizes of the chromosomes. On the basis of arm ratio and total length of the nine chromosomes (fig. 4), chromosomes may be grouped into different categories. Only chromosomes 5 and 9 could be considered submetacentric. The other seven chromosomes had arm ratios smaller than 1.67. Chromosomes 3, 4, and 7 had arm ratios very close to 1 and are considered to be metacentric. The other four chromosomes $(1,2,6$, and 8$)$ may be considered to be meta-submetacentric.

In the chromosome arrangement used for optimum identification of all chromosomes (fig. 1b), the nine chromosomes are grouped into three classes, each with three chromosomes. In the first class

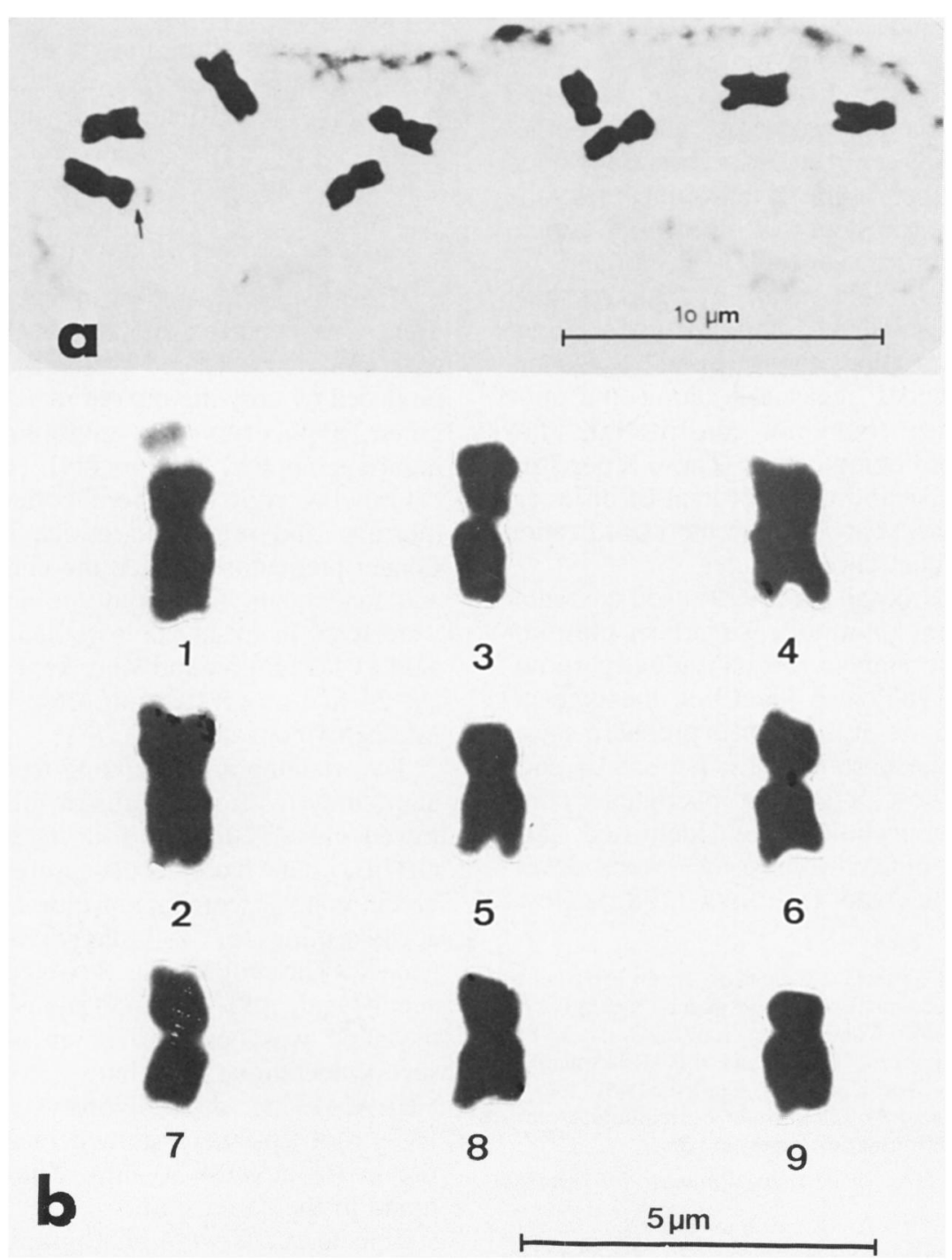

FIG. 1.-Metaphase chromosomes of haploid sugarbeet. $a$, A metaphase cell. $b$, Karyogram of a haploid cell shown in $a$. 
TABLE 1

MEAN CHROMOSOME LENGTH AND ARM RATIO AND THEIR STANDARD ERRORS IN FOUR METAPHASE PLATES IN FIGURES 1 AND 2

\begin{tabular}{|c|c|c|c|c|}
\hline & Chromosome & Total length $(\mu \mathrm{m})$ & Relative length (\%) & Arm ratio $(r)$ \\
\hline $1^{\star 4}$ & & $2.55 \pm .050$ & 12.0 & $1.51 \pm .060$ \\
\hline 2 & & $2.65 \pm .094$ & 12.4 & $1.32 \pm .130$ \\
\hline 3 & & $2.65 \pm .056$ & 12.4 & $1.04 \pm .024$ \\
\hline 4 & & $2.45 \pm .050$ & 11.5 & $1.04 \pm .043$ \\
\hline 5 & & $2.34 \pm .076$ & 11.0 & $1.67 \pm .036$ \\
\hline 6 & & $2.34 \pm .055$ & 11.0 & $1.29 \pm .012$ \\
\hline 7 & & $2.22 \pm .050$ & 10.4 & $1.08 \pm .042$ \\
\hline 8 & $\ldots \ldots \ldots \ldots \ldots$ & $2.12 \pm .031$ & 10.0 & $1.46 \pm .131$ \\
\hline 9 & $\ldots \ldots \ldots \ldots \ldots$ & $1.97 \pm .027$ & 9.3 & $2.08 \pm .092$ \\
\hline
\end{tabular}

${ }^{a}$ The length of the satellite is not included because it varied greatly from cell to cell.
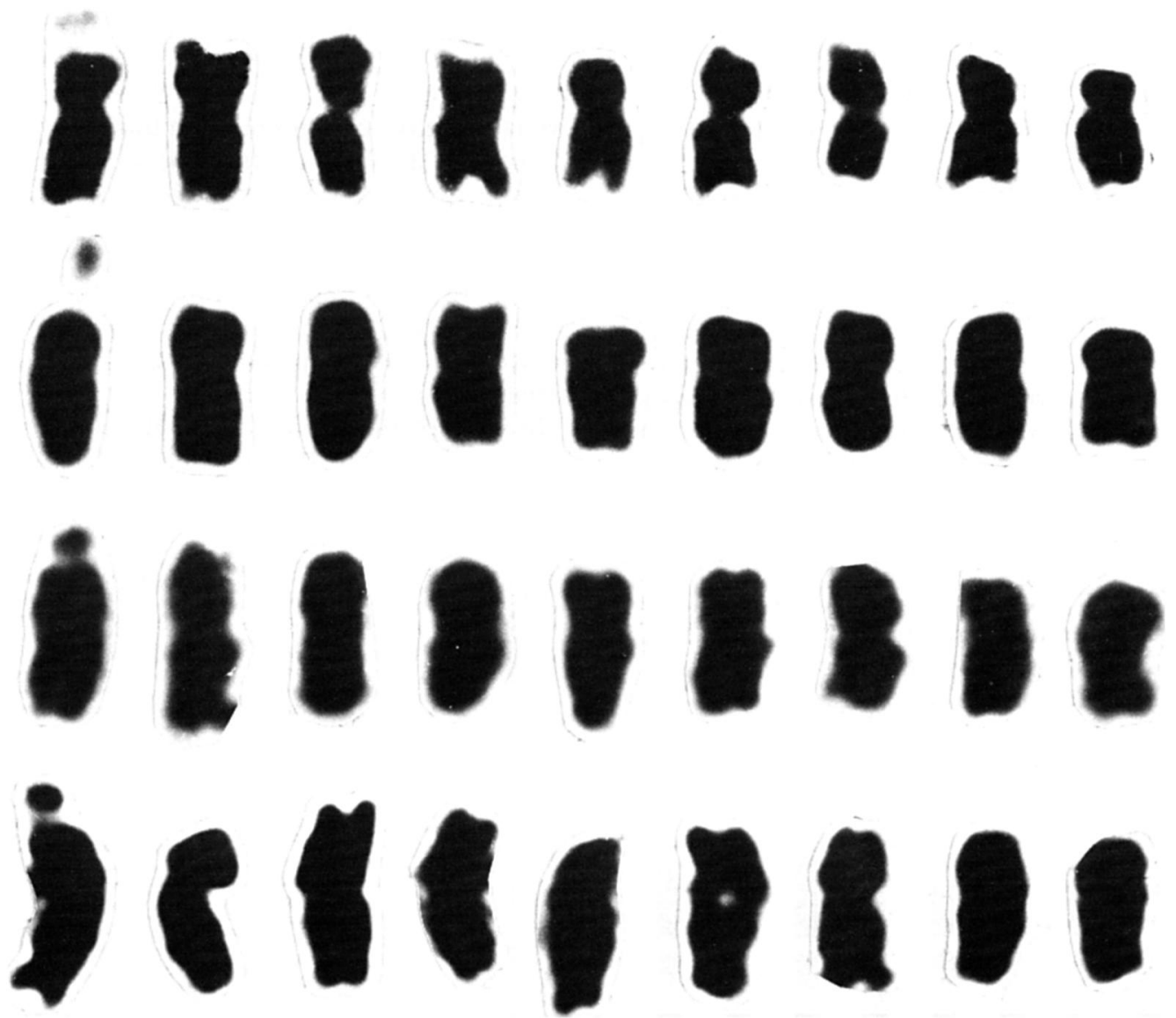

1
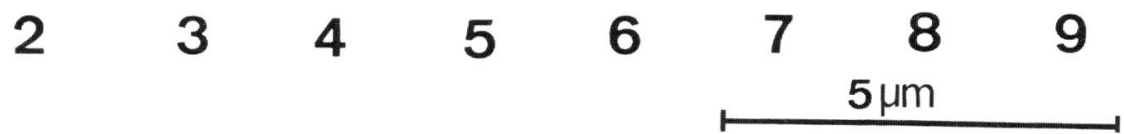

FIG. 2.-Karyogram of metaphase chromosomes from four cells of haploid plants. 
(chromosomes 1, 3, and 4), chromosome 1 is in the first position since it is most easily identified in the complement by its satellite. Chromosomes 3 and 4 are placed together since they are the two large chromosomes with the centromere in the median position; they are similar in length, and there is a high risk of making a reversal-of-order error between the two. The second class consists of chromosomes 3,5 , and 6 ; their centromeres are not in a median position, and they are not the three shortest. Chromosome 2 is the longest of the three. The centromere of chromosome 5 is in a clearly submedian position, and chromosomes 5 and 6 are the same length. Chromosomes 6 and 2 have similar arm ratios, but 6 is shorter than 2 . The three smallest chromosomes are included in the third class: Chromosome 7 has the centromere in a median position and chromosome 8 in a median-submedian position; chromosome 9 is the most submetacentric of the complement.

Although no statistical analysis between cells within a haploid plant or among haploid plants was performed, because of the limited number of selected metaphase cells, no differences in the morphology of the chromosomes were detected (fig. 2).

\section{Discussion}

Haploid plants were selected as the best material for karyotype analysis because the one genome permits a better identification of the nine sugarbeet chromosomes without the confounding effect of homologous chromosomes. Metaphase karyotypes are also best constructed on haploid plants because fewer chromosomes per cell allow an optimum squash and more accurate observation.

BosEMARK and BORMOTOV (1971) and DE JONG and DE BосK (1978) emphasized the problems associated with the continuous variation in the total length and arm ratio of the nine chromosomes. DE JONG and DE BOCK (1978) summarized the causes of variation in the chromosome measurements that

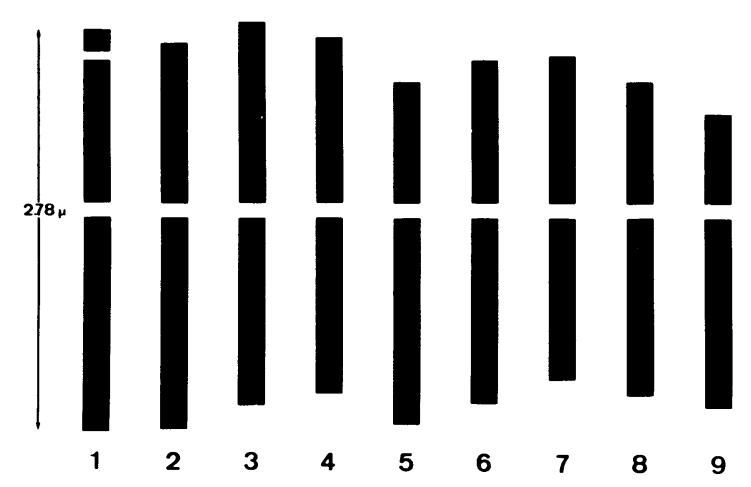

FIG. 3.- Idiogram of sugarbeet chromosomes constructed from karyogram and measurement (table 1) of haploid sugarbeet. increase the probability of reversal-of-order error (MATERN and SiMAK 1968). They listed three causes: (1) variation among repeated measurements of the chromosomes within a cell, (2) differences among cells within one genotype, and (3) variation in chromosome morphology among genotypes. To overcome these sources of error in this study, we used only selected metaphase cells with completely condensed chromosomes on the same focusing plane, as did TJIO and HAgBerg (1951) with barley. Averages of measurements on very enlarged prints by two people were used in this study. Differences were detected between the two analyzed cells of one haploid plant and among the three haploid plants of independent origin.

There still is a considerable probability of reversal-of-order error. MATERN and SIMAK (1968) estimated the minimum difference in total length between two chromosomes to eliminate the probability of this type of error. The risk of reversal error could not be disregarded in their study on the Larix karyotype when the average difference between two chromosomes was less than $11 \%$ of their average length. The maximum difference between two consecutive chromosomes in this study was only $8 \%$ of their average length (table 1). Therefore, no sugarbeet chromosome can be identified simply on the basis of the total length without a considerable probability of reversal-of-order error. Because of this and the small number of analyzed cells, no statistical analysis of the data was performed. Standard errors are given only to show the range of variation.

In spite of these limitations, the nine sugarbeet chromosomes may be satisfactorily distinguished

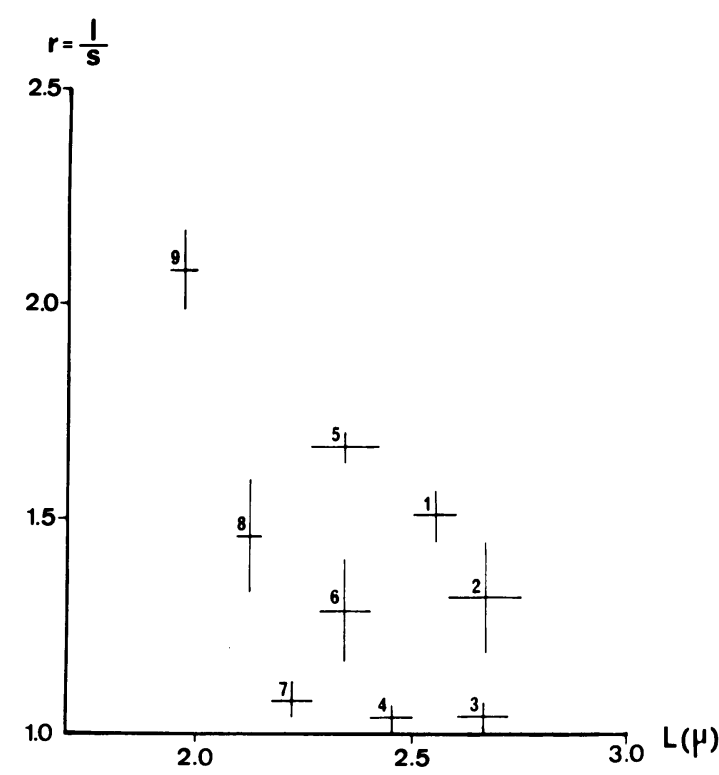

FIG. 4.- Mean chromosome length, $L$, and arm ratio, $r$, ( \pm SE of the mean) of chromosome measurements in table 1 . 
from each other in metaphase cells of good quality. The subgrouping of the nine chromosomes has been useful in identifying the chromosomes. This method has been satisfactorily used in identifying primary trisomic types recently isolated (ROMAGOSA 1982; CiSTUE 1983). By selecting good quality cells, all nine primary trisomic types were cytologically identified, although in a few cases confirmation at the somatic prophase stage was necessary (ROMAGOSA et al., in preparation).

A close correspondence is found between the results in this study and those of BOSEMARK and BORMOTOV (1971). The main difference is found in chromosome 6 of BOSEMARK and BORMOTOV (1971), which seems to correspond to chromosome 4 in this report. Their chromosome 6 was more median than any other in the complement, whereas our chromosome 4 was less median. The small differences between the parameters of the other chromosomes could be a result of (1) misclassification errors in both reports, (2) the different pretreatments used, or (3) as DE JONG and DE Bock (1978) mentioned, different chromosome morphology in the material used. Our results did not agree with those of DE JONG and DE BOCK (1978), who reported that sugarbeet metaphase chromosomes could not be identified on the basis of their relative length and arm ratio. They also mentioned that the satellite of chromosome 1 was not visible in most metaphase plates. These conclusions may be a consequence of limitations imposed by their cytological technique.

\section{LITERATURE CITED}

Bosemark, N. O. 1971. Haploids and homozygous diploids, triploids, and tetraploids in sugar beet. Hereditas 69:193204.

Bosemark, N. O., and V. E. Bormotov. 1971. Chromosome morphology in a homozygous line of sugar beet. Hereditas 69:205-212.

Cistue, L. 1983. Estudios citogeneticos en Beta vulgaris L. Ph.D. diss. Universidad Complutense, Madrid. 198 pp.

DE JoNG, J. H., and T. S. M. DE Bock. 1978. Use of haploids of Beta vulgaris L. for the study of orcein and Giemsa stained chromosomes. Euphytica 27:41-47.

Levan, A., K. FredGa, and A. A. Sandberg. 1964. Nomenclature for centromeric position of chromosomes. Hereditas 52:201-220.
Matern, B., and M. SimaK. 1968. Statistical problems in karyotype analysis. Hereditas 52:201-220.

NaKamura, C., and T. TsuchiYa. 1982. Pachytene chromosome morphology in diploid sugar beet. Z. Pflanzenzucht. 89:229-244.

Romagosa, I. 1982. Primary trisomics in sugar beet. Ph.D. diss. Colorado State University, Fort Collins. $223 \mathrm{pp}$

TuIo, J. H., and A. HagBerg. 1951. Cytological studies on some X-ray mutants of barley. Ann. Aula Dei 2:149-167.

Tsuchiya, T., and C. NaKamura. 1979. Acetocarmine squash method for observing sugar beet chromosomes. Euphytica 28:249-256. 